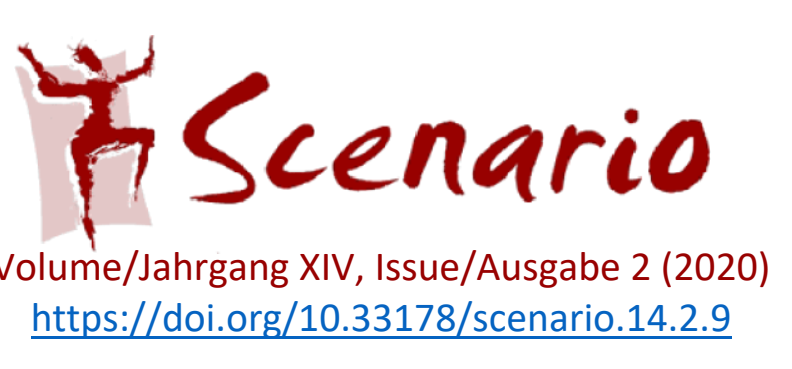

\title{
Rezension: Fremdsprache Deutsch 62 (2020): Performative Didaktik. Berlin: ESV.
}

Almut Küppers

Die in Buchrezensionen vertretenen Ansichten und Meinungen sind die der jeweiligen Rezensentinnen und Rezensenten und reflektieren nicht notwendigerweise die Position von SCENARIO.

„Performative Didaktik“ ist der Titel der Publikation für die Praxis des Deutsch-alsFremdsprache-Unterrichts, um deren Besprechung in SCENARIO ich Ende Mai 2020 gebeten wurde. Die Anfrage erreichte mich somit etwa zehn Wochen nach der gesellschaftlichen Vollbremsung, besser bekannt als „Corona-Lockdown“. Ich litt zu dem Zeitpunkt schon spürbar unter den Folgen von extensiver Bildschirmarbeit mit chronischen Nackenschmerzen, Rücken- und Schulterbeschwerden und träumte täglich von mehr Bewegung. Performatives Lernen ist wichtiger denn je, dachte ich, immer wieder erinnerten mich meine eigenen Schmerzen an die erschreckenden Befunde der US "Inactivity studies“, plakativ zusammengefasst mit: „Sitting is the new smoking“ (Levine 2014) ${ }^{1}$. Konzeption und Planung des Themenheftes zum performativen Lernen hatten sicher viele Monate vor dem Beginn der Pandemie stattgefunden, weswegen ich die Beiträge dieser „Vor-Corona“ Publikation des Goethe-Instituts aus „Nach-Corona“-Perspektive gelesen habe und vorstellen werde.

Fast so, als hätte er den anstehenden Zeitenwandel geahnt, fragt Maik Walter, der Herausgeber dieser lesenswerten Ausgabe der Zeitschrift Fremdsprache Deutsch: „Doch wohin geht der Weg? Die Erfahrung lehrt, dass der Umschwung ins andere Extrem ansteht" (S. 3). Was der Direktor einer der Berliner Volkshochschulen (und einer der aktivsten Vertreter der performativen Lernkultur im Bereich der sprachlichen Bildung) richtig voraussah, ist die Tatsache, dass sich das viel beschworene "didaktische Pendel“ nach langen Zeiten der Verharrung in der Kompetenzorientierung in Bewegung setzen würde. Falsch lag Maik Walter allerdings mit der Richtung, in die das Pendel dann - sehr heftig - ausschlug. Nicht wie damals beim Schreiben des Einführungsbeitrages „Von der Einzigartigkeit des Unterrichtens"

\footnotetext{
${ }^{1}$ Dieses Zitat wird Dr. James Levine zugeschrieben, dem Direktor der Mayo Klinik der Arizona State University. Vgl. auch das Interview mit ihm in Macvean (2014) sowie Küppers (2020: 65ff).
} 
vermutet (und vielleicht erhofft) in Richtung Performanz. Stattdessen schlug das didaktische Pendel mit voller Wucht und flächendeckend in der Unterrichtspraxis ein und zwar als ,digitales Lernen'! Unerwartet, unvorbereitet und zwangsweise wurde als Reaktion auf die Schulschließungen weltweit online Fernunterricht improvisiert. Was in der englischsprachigen Welt als emergency remote teaching bezeichnet wird, setzte voraus, dass Eltern mit der Lernbegleitung ihrer schulpflichtigen Kinder betraut und sie somit erstmals ganz offiziell als unbezahlte Hilfslehrkräfte tätig wurden - und dass internetfähige Endgeräte in ausreichender Anzahl in den Familien vorhanden waren. Die Pandemie wirkte, wie die Publizistin Carolin Emcke (2020) bemerkte, wie ein „Kontrastmittel“, das gesellschaftliche Unzulänglichkeiten, Schwächen und systemische Krankheitsbilder besser sichtbar macht. Zwei bedenkliche - wenn auch nicht neue - Missstände traten durch das virale Kontrastmittel im deutschen Schulsystem noch deutlicher hervor: 1) Corona verschärft die existierende Bildungsungleichheit und 2) die meisten Schulen operier(t)en in digitalen Notstandsgebieten. Seit Sommer 2020 kommt scheinbar kaum eine Tagung oder Veröffentlichung mehr ohne das Attribut ,digital' oder ,online' aus, die digitale Transformation der Schulen ist endlich in (vollem?) Gange und die Digitalisierung des Lernens wird dabei zurecht nicht als eine Notlösung gesehen - im Gegenteil, Bildungsprozesse zu digitalisieren scheint das Gebot der Stunde zu sein, um der explodierenden Vielfalt besser begegnen und auf individuelle Lernbedürfnisse gezielter eingehen zu können und nicht zuletzt um professioneller und nachhaltiger auf erneute lokale Schulschließungen bzw. zukünftige Pandemien reagieren zu können. Ist „Performative Didaktik“ damit aus der Zeit gefallen? Mitnichten! Gerade jetzt und gerade vor dem Hintergrund der Erfahrungen mit (sitzendem) Homeschooling und (einsamen) Fernunterricht vor den Bildschirmen muss die Dimension des Performativen dringend integriert werden in die entstehenden Konzepte zur Digitalisierung der Bildung. Das Themenheft ist daher - um ein Fazit vorwegzunehmen - aktueller und wichtiger denn je!

Das sperrige Wort des „Performativen“ dürfte SCENARIO-Leser*innen geläufig sein. Das Journal for Performative Teaching, Learning and Research führt den Begriff schließlich seit einiger Zeit in seinem Titel. Das Performative an der Performativen Didaktik ist der explizite Bezug zur Welt der Kunst, im Speziellen zur Welt der Bühne, des Theaters, des Tanzes, der Musik und allen weiteren Hybridformen, die in den Zwischenräumen dieser Welten zu finden sind. Gängige Formen ästhetischer Verfahren aus diesen Bereichen werden von der Performativen Didaktik genutzt, um Bildungskontexte zu gestalten und Lernprozesse anzuregen, erläutert Maik Walter in seinem Überblicksbeitrag. Er betont, dass es beim performativen Arbeiten darum geht, „sich mit dem Willen zur ästhetischen Gestaltung auf die Einzigartigkeit einer Handlung einzulassen“ (S. 4) und damit bereit d.h. offen dafür zu sein, 
dass „aus jeder Stunde eine kleine Performance werden (kann)“ (ebd.). Mit welchen Mitteln festgefahrene Routinen im eigenen Unterricht aufgebrochen werden können und wie dieser durch performative Elemente nicht nur bereichert, sondern manchmal vielleicht sogar zu einer einzigartigen Inszenierung werden kann, dazu liefert dieses Heft mit seiner Verortung in der Vorstellung von "Lehren als Kunst" viele Anregungen. Die Zusammenstellung der Praxisbeiträge dient also zunächst dazu, kreative Impulse in die Unterrichtspraxis zu senden. Allen gemeinsam ist dabei die Betonung der Ganzheitlichkeit und des Körpers als wichtige Dimensionen des Lernens sowie der Gruppe als Bezugs- und Erfahrungsraum. Zahlenmäßig dominieren die Beiträge, die im weitesten Sinne der Theater- und Dramapädagogik zugeordnet werden können: Claudia Bartholomeycziks liefert in ihrem Beitrag „Von der Idee zur Aufführung" eine Art Blaupause, wie man in einer Lerngruppe ein komplettes Theaterstück entwickeln und inszenieren kann. Wem eine ganze Aufführung eine ,Nummer zu groß' ist, der/die sei verwiesen auf den Beitrag von Birgit Bader "Vom Text zum Spiel“, in dem es um Rollen(spiel)arbeit geht, durch die ein Text oder Textpassagen im Unterricht performativ zum Leben erweckt werden können. Und wem auch Texte und Szenen noch zu umfangreich erscheinen, der/die fängt vielleicht auf der Satzebene an performativ zu arbeiten. Doreen Bryant und Sarah Unger liefern dazu ein anregendes Beispiel und zeigen in ihrem Beitrag „Sich Grammatik erspielen“, wie sich Lernende durch den Einsatz von dramagrammatischen Mitteln Relativsätze erarbeiten, die dadurch erfahrbar und leichter zu verstehen sind. Das sogenannte „Klassenzimmerstück“ wird im Beitrag von Lasse Scheiba und Maik Walter vorgestellt. Dabei wird das Klassenzimmer zur Theaterbühne für andere: Schauspieler oder Theaterpädagogen werden eingeladen, die in und/oder mit der Klasse ein Stück inszenieren, häufig zu einem brisanten Thema wie Cybermobbing, Rassismus oder sexuellem Missbrauch. Das Stück, das hier vorgestellt wird, heißt "Mein ziemlich seltsamer Freund Walter" und handelt von einem Außenseiter und Ausgrenzung.

Zwei Beiträge nutzen die Offenheit und Kraft der Bilder - bewegt oder fixiert - als Grundlage für performative Spracharbeit. Sigrid Unterstabs Perspektive liegt in dem Beitrag „Was Bilder uns geben - was wir Bildern geben“ auf dem Zusammenspiel von Sprache, Körper und Fotographie, mit welchen sie einen Kontext schafft für künstlerisches Arbeiten mit Jugendlichen und als Ausgangspunkt für selbstreflexive Prozesse. Dragan Miladinović knüpft an den multimodalen Sehgewohnheiten junger Menschen an und nutzt in der von ihm vorgestellten Unterrichtssequenz einen sehr kurzen Kurzfilm und seine Suggestivkraft. In seinem Beitrag „Filme performativ in Szene setzen“ stellt er dar, wie eine Film-Geschichte, die ausschließlich durch die Sprache der Bilder erzählt wird, mit Hilfe kleiner dramapädagogischer Formen wie dem Heißen Stuhl in ihrer Multiperspektivität ausgeleuchtet und sehr 
schüler*innenorientiert für kreative Spracharbeit genutzt werden kann. Sprachenlernen durch die magnetisierende Wirkung der Erzählkunst zu beflügeln, ist das Metier von Kristin Wardetzky. In ihrem spannenden Hintergrund-Beitrag basierend auf langjährigen Erfahrungen in Willkommensklassen wird deutlich, dass die Kunstform des Geschichtenerzählen hochgradig performativ ist: Mit Körpereinsatz und der Stimme als viel-seitigem Instrument wird eine magische Verbindung zu den Zuhörenden aufgebaut, diese werden förmlich in die Geschichte hineingezogen und dadurch zu Co-Autor*innen.

Die Printausgabe des Themenheftes Performative Didaktik wird auf der Webseite ${ }^{2}$ der Zeitschrift Fremdsprache Deutsch ergänzt durch vier komplette und ebenfalls empfehlenswerte open-access Beiträge, die sich mit Erzählkunst (Freya Conesa) und Sprechtraining mit Theater-Elementen im Anfänger*innenunterricht (Anja Schürmann) beschäftigen sowie mit performativem Sprachenlernen durch kreative Textarbeit „von Rappen bis Einfrieren“" (Claudia Böschel) und Theaterelementen im Anfänger*innenunterricht (Ulla Krawczyk). Besonders erwähnenswert ist hier eine Sammlung mit wertvollen Zusatzmaterialien zum Beitrag von Claudia Bartholomeyczik in der Printausgabe mit dem Fokus Stückentwicklung fürs Theater von der Idee bis zur Aufführung. Die Auflistungen mit Hinweisen aus der Feder einer erfahrenen Theaterpädagogin z.B. für "Szenenproben“, „Figurenfindung“, „Rollenbiographien entwickeln“, „Bühnenpräsenz“ oder „Zeitplan für Aufführungen " sind enorm hilfreich - insbesondere für Lehrkräfte, die sich vielleicht erstmalig an eine größere Inszenierungsform heranwagen wollen.

Die vielfältigen Praxisbeiträge halten einerseits für Neulinge und erfahrene/re Kolleg*innen gleichermaßen Inspirierendes bereit. Andererseits bietet das Themenheft aber auch einen Einstieg in die Beschäftigung mit dem theoretischen Fundament der Performativen Fremdsprachendidaktik. Empirische Begründungen erhält diese durch die Befunde der Neurowissenschaften, auf die Michaela Sambanis in ihrem Beitrag „Probier's mal mit Beweglichkeit" eingeht. Sie zeigt, dass bewegtes Lernen nicht nur nachhaltig/er ist, sondern auch Gruppenprozesse fördert und in Form von kleineren Theaterübungen punktuell und unkompliziert in den Unterricht integriert werden kann. Abschließend sei auf Manfred Schewes kurze Einführung „Unterricht als Kunst“ verwiesen. Hier formuliert „der Wegbereiter der Performativen Fremdsprachendidaktik“ (Walter, S. 7) einige grundlegende theoretische Perspektiven zum Verhältnis von Wissenschaft und Kunst in der Fremdsprachenvermittlung und entsprechend auch in der Ausbildung zukünftiger Lehrkräfte. Zunächst konstatiert der „Nestor der Dramapädagogik im Bereich Deutsch als Fremdsprache“ (ebd.) mit Blick auf

${ }^{2}$ https://www.fremdsprachedeutschdigital.de/webangebot.html 
derzeitige Lehramtsstudiengänge, dass „eine ausschließliche wissenschaftliche Beschäftigung mit Aspekten des Lehrens und Lernens von Sprache, Literatur, Kultur nicht mehr angemessen ist, sondern neben der Wissenschaft auch die Kunst eine wichtige Rolle in der Fremdsprachenvermittlung spielen sollte" (S. 9). Er begründet diese Forderung u.a. mit dem Verweis auf die ökologischen und gesellschaftlichen Herausforderungen des 21. Jahrhunderts, für die die (jetzt heranwachsenden jungen) Menschen kreative Antworten finden müssen und bezieht sich dabei auf alte Forderungen der UNESCO aus den Jahren 2006 und 2010, die Künste in der Bildung dringend zu stärken.

Die Künste in der Bildung stärken? Auf welche Fächer wurde sich während der Schulschließungen im Frühjahr 2020 konzentriert? Etwa Musik, Tanz, Sport, Zeichnen, Malen, Theater oder Darstellendes Spiel? Die meisten Schulen fokussierten auf die sogenannten ,Hauptfächer': Deutsch, Mathematik und Englisch. Vor allem für diese drei Fächer wurden Arbeitsaufträge häufig in bewährter ,Arbeitsblatt"-Form als umfangreiche PDF-Dateien per EMail an die Abnehmer*innen versendet (aka „Death by Worksheet“). In Deutschland konnten nur wenige Schulen auf voll etablierte Kommunikationsplattformen wie Moodle zurückgreifen, über die Homeschooling zu einer anregenden und auch kollaborativen Erfahrung wurde bzw. hätte werden können. Das virale Kontrastmittel Corona deutete hier auf einen weiteren besorgniserregenden Befund: Das, was als sogenanntes „digitales Lernen“ stattfand (PDFs per E-Mail versenden) zeichnete sich zudem durch erstaunliche Ideen- und Kreativlosigkeit aus. Das traf und trifft sicherlich nicht für alle Schulen in gleichem Maße zu, denn in einigen Schulen wurde der sich öffnende Raum in die digitale Bildung geradezu vorbildlich genutzt, indem sich kreative Kolleg*innen fanden, die zusammen unkonventionelle Wege beschritten ${ }^{3}$. Das von Manfred Schewe konstatierte Defizit der Kunstlosigkeit von Unterricht konnte von vielen Eltern während der Schulschließungen also tatsächlich besichtigt werden. Einer der schärfsten und populärsten Kritiker bestehender Bildungssysteme, Sir Ken Robinson, unterstellte über Jahre hinweg: „schools kill creativity“4. Er plädierte für nicht weniger als eine „Bildungsrevolution“ und forderte den Status der Kreativität massiv aufzuwerten: Konformität, die Bildungsmaxime der Nationalstaaten im 19. Jahrhundert,

\footnotetext{
${ }^{3}$ Als ein Beispiel dient hier eine Gesamtschule in Hessen, die mit sogenannten "Challenges“ ihre Klassen dazu aufrief, gegeneinander im Wettbewerb um die besten Gemeinschaftsergebnisse anzutreten. Sport war dabei immer eine Teilaufgabe, aber eben auch die Fächer Kunst, Biologie oder Geschichte: https://arslangen.de/index.php/schulleben/artikel-aus-der-schule/688-ars-klassen-challenge-macht-alle-mit (letzter Zugriff: 17.11.2020)

${ }^{4}$ Der TED-Talk von 2006 des mittlerweile verstorbenen Professors für Kunst ist einer der populärsten überhaupt. https://www.ted.com/talks/sir ken robinson do schools kill creativity?referrer=playlist-

the most popular talks of all In seinem TED-Talk von 2010 stellte er explizit einen Zusammenhang zwischen der ersten und zweiten Klimakrise (der in der Schule) her: https://www.youtube.com/watch?v=r9LelXa3U I (letzter Zugriff: 17.11.2020)
} 
müsse dringend durch das Paradigma der Kreativität abgelöst werden (vgl. Robinson \& Aronica 2016: 238). Ähnlich sieht es - übrigens nicht erst seit den Erfahrungen während der Corona bedingten Schulschließungen - der Rat für Kulturelle Bildung in Deutschland. In einer gerade erschienenen Denkschrift, die sich an Entscheidungsträger*innen im Bildungsbereich richtet, heißt es: „Der Kulturort Schule muss und wird sich deutlich, möglicherweise fundamental wandeln" (Rat für Kulturelle Bildung 2020: 4). Für die Autor*innen ist dabei evident, dass der kulturellen Bildung - und damit sind explizit die Künste gemeint - bei der Integration digitaler und analoger Formen von schulischer Praxis eine wachsende Bedeutung zukommen wird (ebd.). ${ }^{5}$

Wie es gelingen kann, die Künste in den etablierten Fächern der (fremd-) sprachlichen Bildung zu integrieren und damit die im Raum stehenden dringend notwendigen bildungspolitischen Forderungen nach mehr kultureller Bildung umzusetzen, um die Schule in einen Kulturort zu verwandeln, davon kann man sich durch die Lektüre dieses gelungenen Themenheftes ein Bild machen. An einigen Stellen sind die Bezüge zur digitalen Kunst und digitalen Formen der performativen Spracharbeit schon explizit sichtbar, wie etwa in dem Beitrag von Claudia Bartholomeyczik: Während der Entwicklung eines Theaterstückes erleichtern digitale Werkzeuge nicht nur die Administration und Kommunikation, wenn z.B. Smartphones genutzt werden, um sich spontan abzusprechen oder Kostümvorschläge zu brainstormen. Immens wertvoll für die kreative Zusammenarbeit war z.B. auch eine Cloud-Plattform, die von Gruppenmitgliedern genutzt wurde, um parallel am Herzstück der Aufführung, der Textgrundlage zu arbeiten. Der Ausschlag des didaktischen Pendels Richtung digitales Lernen ist keinesfalls (zwangsläufig) am Performativen verbeigerauscht. Im Gegenteil! Viele der populären Online-Werkzeuge wie Padlet, Bookcreator oder Mentimeter verfügen über großes performatives Potenzial für künstlerisches, kreatives und kooperatives Arbeiten und Lernen in Gruppen. Die Risiken und Nebenwirkungen von stundenlangem Sitzen - egal ob im Klassenraum oder am Bildschirm - sind hinlänglich bekannt (s.o.) mit negativen Begleiterscheinungen nicht nur für Körper und Seele, sondern vor allem auch für das sozialemotionale Lernen auf der Beziehungsebene. Insofern ist zu hoffen, dass nicht nur der analoge Unterricht in den Präsenzphasen, sondern vor allem auch das ergänzende digitale Lernen durch die Performative Didaktik in Bewegung kommt. Aktuelle Themenhefte einschlägiger

\footnotetext{
${ }^{5}$ Die konkreten Empfehlungen des Rats für Kulturelle Bildung beziehen sich dabei auf eine Unterrichtsgarantie in den künstlerischen Fächern, eine Abdeckung der ästhetischen Bereiche im Curriculum und um einen fächerübergreifenden Einsatz ästhetischer Prinzipien. Damit einhergehen muss die Aufwertung des kulturellen Angebots und eine Verstärkung von Bildungspartnerschaften und -kooperationen sowie als Voraussetzung dessen, eine entsprechende Qualifizierung für Lehrkräfte und andere in der Schule tätigen Professionellen (2020: $57 \mathrm{ff}$.)
} 
Fachzeitschriften führen derzeit viel „Digitales“ im Titel. Neue Synergien zu schaffen und die noch nie gekannte Fülle an ausgezeichneten Inhalten, Werkzeugen und Methoden der Onlineund Offline-Welten zu nutzen, um die Qualität schulischer Bildung im 21. Jahrhundert durch Kunst und Kreativität in allen Dimensionen anzureichern und zu verbessern, darum muss es gehen, wenn Digitales und Performatives in zeitgemäßen Bildungskonzepten zukünftig explizit zusammengedacht - und gebracht werden.

Bibliografie

Emcke, Carolin (2020): Politisch-persönliche Notizen zur Corona-Krise. Woche 1 / 25. März. In: SZ.de https://projekte.sueddeutsche.de/artikel/politik/corona-krise-journal-in-zeiten-der-pandemiee584987/ [letzter Zugriff: 20.10.2020]

Fremdsprache Deutsch 62 (2020): Performative Didaktik. ESV, https://doi.org/10.37307/j.2194$\underline{1823.2020 .62}$

Macvean, Mary (2014): 'Get up!' or Lose Hours of Your Life, Scientist says. The Los Angeles Times, July 31 .

Küppers, Almut (2020): Mehrsprachigkeit in Bewegung. Dramapädagogik und mehrsprachiges Lernen. In: Stolarczyk, Barbara \& Merkelbach, Christoph (Hrsg.): Herkunftssprachen - Polnisch, Russisch und Türkisch in der interkulturellen und mehrsprachigen Gesellschaft in der Bundesrepublik. Düren: Shaker-Verlag, S. 61-72

Rat für Kulturelle Bildung (Hrsg.) (2020): Kulturort Schule. Bildungspolitische Handreichung. Reihe Auf den Punkt I/III https://www.rat-kulturellebildung.de/fileadmin/user upload/RFKB Kulturort Schule.pdf [letzter Zugriff: 20.10.2020]

Robinson, Ken \& Aronica, Lou (2016): Creative Schools. The Grassroots Revolution That's Transforming Education. New York: Penguin Books 